\title{
Representação da Diversidade de Componentes Latentes em Exercícios de Programação para Classificação de Perfis
}

\author{
Márcia G. de Oliveira ${ }^{1}$, Nátaly A. Jiménez Monroy $^{2}$, Paula Daher Ximenes ${ }^{2}$, Elias Oliveira ${ }^{1}$ \\ ${ }^{1}$ Programa de Pós-Graduação em Informática (PPGI) \\ Universidade Federal do Espírito Santo \\ Vitória - ES - Brasil \\ ${ }^{2}$ Departamento de Estatística \\ Universidade Federal do Espírito Santo \\ Vitória - ES - Brasil \\ clickmarcia@gmail.com, elias@acm.org
}

\begin{abstract}
Using the factorial analysis technique, a set of factors or latent components can be discovered from quantitative variables to characterize programs written by students. Representing each program by a set of latent components, we call this representation of student profile. In order to classify students profiles, this paper proposes a non-random selection method based on Graph Clustering to select representative profiles from the diversity of latent components to build a training set for classification models. The results achieved demonstrate that the combination of factorial analysis with Graph Clustering improves outcomes of profiles classification.
\end{abstract}

Keywords: Factorial analysis, Programming Learning, Classification of Profiles, Graph Clustering.

Resumo. Através da técnica de análise fatorial é possível descobrir um conjunto de fatores ou componentes latentes a partir de variáveis quantitativas que caracterizam programas desenvolvidos por alunos. Ao representar cada programa por um conjunto de componentes latentes, chamamos essa representação de perfil de estudante. Com o objetivo de classificar perfis de estudantes, este trabalho propõe um método de seleção não-aleatória baseado em Clustering em Grafo para selecionar perfis representativos da diversidade de componentes latentes como conjunto de treino de modelos de classificação. Os resultados alcançados demonstram que a combinação da técnica de análise fatorial com Clustering em Grafo melhora os resultados de classificação de perfis.

Palavras-chave: Análise fatorial, Aprendizagem de programação, Classificação de perfis, Clustering em Grafo.

\section{Introdução}

A programação de computadores é um conhecimento que demanda extensa prática de exercícios para ser de fato aprendido. No entanto, essa prática deve ser assistida por um professor de forma que seus feedbacks possibilitem a evolução dos estudantes em seu processo de aprendizagem. A prática assistida de programação, porém, torna-se um 


\section{CBIE-LACLO 2015}

Anais do XXVI Simpósio Brasileiro de Informática na Educação (SBIE 2015)

desafio quando se considera o grande esforço de professores para avaliar individualmente muitos exercícios, especialmente em cursos massivos [Pieterse 2013].

Embora muitas propostas de tecnologias de avaliação automática de exercícios de programação tenham sido desenvolvidas [Oliveira and Oliveira 2015], ainda estamos longe de uma tecnologia que de fato avalie como um professor avalia.

Podemos, no entanto, criar estratégias semi-automáticas de avaliação que possibilitem qualificar a aprendizagem de programação aplicando muitos exercícios, sem necessariamente atribuir uma nota exata para cada exercício. Uma solução para isso seria, por exemplo, extrair de programas escritos por alunos, atributos que nos permitam representar e classificar perfis por níveis de aprendizagem. Nesse caso, a ideia seria estabelecer faixas de scores representando classes de perfis de estudantes.

Seguindo essa ideia, o trabalho de [Oliveira et al. 2014] propõe uma estratégia de mapeamento e classificação de perfis de alunos a partir de programas em Linguagem C. O mapeamento de perfis é uma representação de programas por variáveis chamadas componentes de habilidades medidas pela contagem de atributos de código-fonte e por flags indicadores de execução correta. Já as componentes latentes representam em fatores as relações entre as componentes de habilidades. Através de uma seleção aleatória de amostras representadas por componentes latentes, um classificador é treinado e, a partir dessa aprendizagem, classifica outras amostras por faixas de scores de classes.

Este trabalho estende a proposta de classificação de perfis de [Oliveira et al. 2014] ao propor um método de seleção não-aleatória de amostras de treino para classificação de perfis através da combinação das técnicas de análise fatorial e de Clustering em Grafo. A análise fatorial é utilizada para redução de dimensionalidade e o Clustering em Grafo, para a seleção de amostras que representem a diversidade de componentes latentes.

Integrado a sistemas de sistemas de apoio à prática de programação como o $\mathrm{PCo}$ digo [Oliveira et al. 2015], o método de seleção e classificação de perfis pode ser utilizado por professores para fornecer feedbacks mais imediatos para seus alunos.

Para apresentar o método proposto, este trabalho foi organizado conforme a ordem a seguir. Na Seção 2, descrevemos as técnicas utilizadas e os trabalhos relacionados. Na Seção 3, apresentamos a arquitetura de funcionamento da classificação de perfis. Na Seção 4, detalhamos os procedimentos, os materiais e os resultados de aplicação do método. $\mathrm{Na}$ Seção 5, concluimos com as considerações finais e propostas de trabalhos futuros.

\section{Fundamentação Teórica}

O método desenvolvido neste trabalho combina as técnicas de análise fatorial e de Clustering em Grafo para seleção não-aleatória de amostras de treino de modelos de classificação de perfis. A ideia desse método é baseada na proposta de representação e classificação de perfis de [Oliveira et al. 2015] e [Oliveira et al. 2014] e na estratégia de clusterização de plágios em exercícios de programação de [Moussiades and Vakali 2005].

\subsection{A Análise Fatorial}

A análise fatorial é uma técnica de análise multivariada que consiste na redução da dimensionalidade de um conjunto de dados, encontrando grupos homogêneos de variáveis a partir de um grande número de variáveis. Esses grupos homogêneos, também chamados 


\section{CBIE-LACLO 2015}

Anais do XXVI Simpósio Brasileiro de Informática na Educação (SBIE 2015)

de fatores, são formados por variáveis muito correlacionadas entre si. O ideal é que os fatores sejam independentes uns dos outros. Portanto, a análise fatorial é uma técnica que busca explicar ao máximo a informação contida nos dados, usando a menor quantidade possível de dimensões [Oliveira et al. 2014].

Frequentemente as influências dos $k$ fatores costumam ser divididas em comuns e específicas. Por exemplo, há fatores altamente informativos que são comuns para todas as $p$ componentes de $\mathbf{X}$ e fatores que são específicos apenas para algumas componentes. Nesse caso, a matriz $\mathbf{X}$ pode expressar-se como

$$
\mathbf{X}=\mathbf{Q F}+\mathbf{U}+\mu
$$

onde $\mathbf{Q}$ é uma matriz $(p \times k)$ de coeficientes (não-aleatórios) dos fatores comuns $\mathbf{F}$ (de dimensão $(k \times 1))$, $\mathbf{U}$ é uma matriz $(p \times 1)$ dos fatores (aleatórios) específicos e $\mu$ é o vetor de médias de $\mathbf{X}$.

Assumindo que um modelo fatorial com $k$ fatores foi encontrado razoável, isto é, a maioria das variações das $p$ variáveis em $\mathbf{X}$ foram explicadas por $k$ fatores fixos, podemos interpretar os fatores $F_{l}, l=1, \ldots, k$ calculando suas correlações com as variáveis $X_{j}$, $j=1, \ldots, p$, para obter a matriz $\mathbf{P}_{X F}$. Essa correlação é dada por

$$
P_{X F}=D^{-1 / 2} Q
$$

onde $D=\operatorname{diag}\left\{\sigma_{X_{1} X_{1}}, \ldots, \sigma_{X_{p} X_{p}}\right\}$, sendo $\sigma_{X_{j} X_{j}}$ a variância de $X_{j}$. Usando essa informação podemos determinar quais das variáveis originais $X_{1}, \ldots, X_{p}$ influenciam nos fatores não observados $F_{1}, \ldots, F_{k}$ [Anderson 1984, Morrison 1990].

\subsection{Clustering em Grafo}

Os grafos são estruturas formadas por um conjunto de vértices e de arestas. Em um grafo, os vértices são nós ou representações de objetos e as arestas são conexões entre pares de vértices. O Clustering em Grafo é a tarefa de agrupar os vértices de um grafo em clusters de forma que haja muitas arestas dentro dos clusters e poucas entre eles [Schaeffer 2007].

Um corte de grafo é o particionamento dos vértices desse grafo em dois clusters. As arestas de corte são aquelas que possuem vértices em dois clusters. O mínimo corte consiste em achar um particionamento em que a soma dos pesos das arestas de corte seja o mínimo possível.

Um exemplo de Clustering em Grafo é apresentado na Figura 1, onde $C l_{1}, C l_{2}$ e $\mathrm{Cl}_{3}$ são os clusters separados pelos cortes mínimos $P_{1}$ e $P_{2}$.

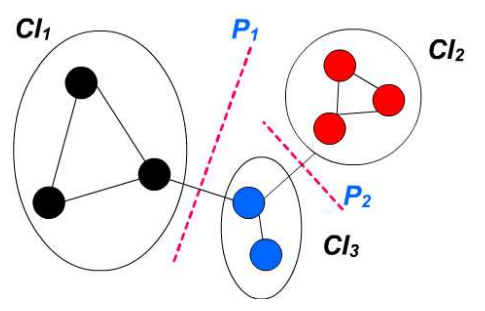

Figura 1. Clustering em Grafo

Neste trabalho, utilizamos o algoritmo de Clustering em Grafo do software Cluto [Karypis 2003]. A medida de similaridade escolhida foi o cosseno. Já os parâmetros de 
corte utilizados foram o edgeprune $=0.7$ e o vtxprune $=0.4$. O primeiro parâmetro separa certas arestas do grafo dos vizinhos mais próximos que tendem a conectar vértices pertencentes a diferentes clusters. Já o segundo parâmetro separa certos vértices do grafo de vizinhos mais próximos que não se agrupam em qualquer cluster.

\subsection{Trabalhos Relacionados}

O PCodigo, um sistema de apoio à prática assistida de programação desenvolvido por [Oliveira et al. 2015], representa perfis de estudantes a partir de programas em Linguagem C. Esses perfis são vetores cujas dimensões são formadas a partir da contagem de atributos da Linguagem $\mathrm{C}$ e por flags indicadores de compilação e de execução.

Para resolver o problema de se obter scores que melhor representem as classes de perfis de estudantes no PCodigo, [Oliveira et al. 2014] propõem um método de seleção aleatória de amostras de cada cluster gerado pelo algoritmo Bissecting-kmeans [Karypis 2003]. Em seguida, os scores dos demais perfis de cada cluster são preditos e uma classe de nível de apredizagem é associada a cada perfil.

O PDetect é um sistema que faz análise e clusterização de plágios em exercícios de programação [Moussiades and Vakali 2005]. Para isso, o PDetect recebe códigos-fontes, mapeia-os em um conjunto de tokens de uma linguagem de programação, identifica os pares suspeitos de plágios e os reúne em clusters, formados a partir de um parâmetro de corte, que é o Coeficiente de Jaccard [Moussiades and Vakali 2005].

O trabalho de [Moussiades and Vakali 2005] utilizou o Clustering em Grafo para separar agrupamentos de plágios de exercícios de programação. Seguindo essa ideia para classificar os perfis gerados pelo PCodigo, estendemos a proposta de [Oliveira et al. 2014] combinando a análise fatorial e o Clustering em Grafo para seleção não-aleatória dos conjuntos de treino dos modelos de classificação de perfis de estudantes de programação.

\section{Método de Representação da Diversidade de Componentes Latentes}

A Figura 2 ilustra a arquitetura do método de representação, de seleção e de classificação de perfis deste trabalho. O módulo de Mapeamento de Perfis recebe programas de computador em Linguagem $\mathrm{C}$ e os transforma em vetores cujas dimensões são chamadas de componentes de habilidades $\left(C_{i}\right)$. Cada componente de habilidade é quantificada pela frequência de ocorrência de palavras reservadas, símbolos, operadores e funções da Linguagem $\mathrm{C}$ ou por flags que indicam se um programa funcionou ou não [Oliveira et al. 2014].

O módulo de Análise Fatorial, conforme a Figura 2, redimensiona os perfis gerados pelo módulo de Mapeamento de Perfis extraindo as componentes latentes através das relações entre as componentes de habilidades $C_{i}$ [Oliveira et al. 2014].

No módulo de Seleção de Diversidade, todos os perfis representados pelas componentes latentes $F_{j}$ são submetidos ao algoritmo de Clustering em Grafo para seleção não-aleatória de treino do modelo de classificação de perfis. Em seguida, é executada a função de Separação Treino/Teste que seleciona as amostras de treino do modelo de classificação e as amostras que serão classificadas por esse modelo.

O conjunto das amostras de treino, isto é, o conjunto $R_{D}$ da Figura 2, é formado pelas amostras que não foram clusterizadas pelo algoritmo de Clustering em Grafo, isto é, pelas amostras que não se agrupam a qualquer dos $k$ clusters ou cujos vértices estejam 


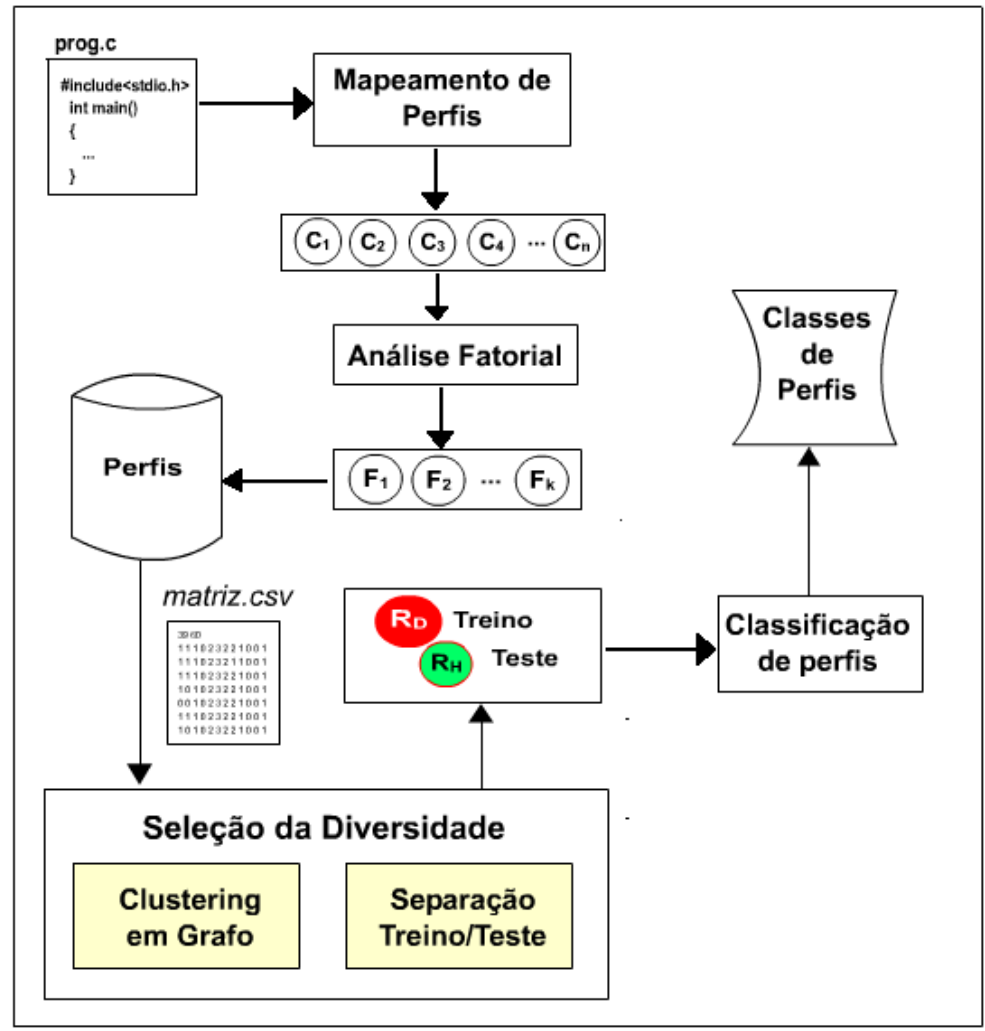

Figura 2. Mapeamento, seleção e classificação de perfis

entre dois clusters. O conjunto $R_{D}$ representa, portanto, as amostras com maior diversidade das componentes latentes. Já o conjunto $R_{H}$ das amostras a serem classificadas é formado pelos clusters gerados pelo algoritmo de Clustering em Grafo. Esses $k$ clusters são agrupamentos homogêneos das amostras com similaridade acima de $70 \%$ entre si.

A saída do módulo de Seleção de Diversidade são dois arquivos contendo perfis de treino e de teste, respectivamente. O Treino é pré-classificado por um professor e é utilizado na aprendizagem do modelo de classificação. Já o Teste contém as amostras que serão classificadas pelo módulo de Classificação de Perfis.

No módulo de Classificação de Perfis, utilizamos modelos de regressão linear para predição dos scores que representam as classes de cada perfil do conjunto de teste. Os valores dos scores de referência das Classes de Perfis, em uma escala de 0 a 5, são 0.5 (ruim), 2.0 (regular), 2.75 (médio), 4.0 (bom) e 5.0 (Excelente). Dessa forma os estudantes são classificados por scores iguais ou próximos a esses valores.

Em resumo, a metodologia deste trabalho visa aproximar-se o máximo possível do score de classe atribuído pelo professor a um exercício de programação.

\section{Experimentos e Resultados}

A experimentação do método deste trabalho foi realizada através dos seguintes passos:

1. Separação das bases experimentais

2. Representação das bases em matrizes

3. Aplicação da análise fatorial nas bases experimentais 


\section{CBIE-LACLO 2015}

Anais do XXVI Simpósio Brasileiro de Informática na Educação (SBIE 2015)

4. Seleção dos conjuntos de treino dos modelos de classificação

5. Classificação de Perfis

6. Apresentação dos resultados

Os procedimentos de cada um desses passos de experimentação são descritos em detalhes nas subseções a seguir.

\subsection{Separação das Bases}

Para a realização dos experimentos deste trabalho, utilizamos duas bases, a Base- $A$ e a Base-B. A Base-A é formada por 100 exemplos de programas em Linguagem C desenvolvidos por alunos em resposta a um exercício de programação. A Base-A foi a mesma base utilizada nos experimentos de [Oliveira et al. 2014].

A Base- $B$, por sua vez, foi gerada em condições controladas, pois as amostras obtidas são programas em Linguagem $C$ desenvolvidos por alunos para uma questão de prova aplicada em uma turma de programação de uma universidade.

A Base-B é formada por 39 amostras de programas e é considerada difícil por ser uma base pequena e com alta diversidade de soluções.

A especificação do exercício das duas bases é a mesma e consiste em construir um programa em Linguagem C para identificar o campeão e o vice-campeão de um campeonato de futebol a partir das pontuações obtidas por três times.

\subsection{Representação das bases}

As amostras de programas da Base-A e da Base-B foram representadas em vetores com 60 dimensões chamadas componentes de habilidades, que representam a frequência de ocorrência de palavras-chave, operadores, funções e estruturas da Linguagem $\mathrm{C}$ e flags que informam se um programa funcionou (valor 1) ou não (valor 0) [Oliveira et al. 2015]. Em seguida, os vetores da Base-A foram reunidos na matriz $M_{100 \times 60}$ e os da Base-B, na matriz $N_{39 \times 60}$.

\subsection{Aplicação da Análise Fatorial}

A técnica de análise fatorial foi aplicada nas bases experimentais deste trabalho utilizando o método das componentes principais com Rotação Varimax e adotando como critério de decisão do número de fatores os autovalores maiores que 1 e com base no scree-plot (Figura 3). O processo de análise fatorial da Base-A e a seleção de seus doze fatores são explicados em detalhes por [Oliveira et al. 2014]. Neste trabalho, apresentamos apenas o processo de análise fatorial da Base- $B$.

Das 60 variáveis (ou componentes de habilidades) da Base-B, 29 tiveram valores iguais a zero em todas as amostras e nove variáveis foram utilizadas somente uma vez pelos alunos. Essas variáveis foram retiradas da análise, restando apenas 22 variáveis.

Um modelo com cinco fatores mostrou-se adequado explicando $81 \%$ da variabilidade dos dados originais. Na Tabela 1, são apresentados os autovalores e as porcentagens da variabilidade explicada e acumulada.

A Figura 3 mostra o scree plot, isto é, a relação entre autovalores e fatores, utilizado para determinar a quantidade de fatores. Observe que, a partir do Fator 5, o autovalor 
CBIE-LACLO 2015

Anais do XXVI Simpósio Brasileiro de Informática na Educação (SBIE 2015)

\begin{tabular}{llllll}
\multicolumn{7}{c}{ Autovalores e Variabilidade } \\
\hline & Fator 1 & Fator 2 & Fator 3 & Fator 4 & Fator 5 \\
\hline Autovalores & 8,449 & 4,762 & 1,661 & 1,560 & 1,382 \\
Variabilidade & 38,406 & 21,647 & 7,550 & 7,092 & 6,284 \\
Variabilidade Acumulada & 38,406 & 60,053 & 67,602 & 74,694 & 80,978 \\
\hline
\end{tabular}

Tabela 1. Autovalores e porcentagem da variabilidade explicada

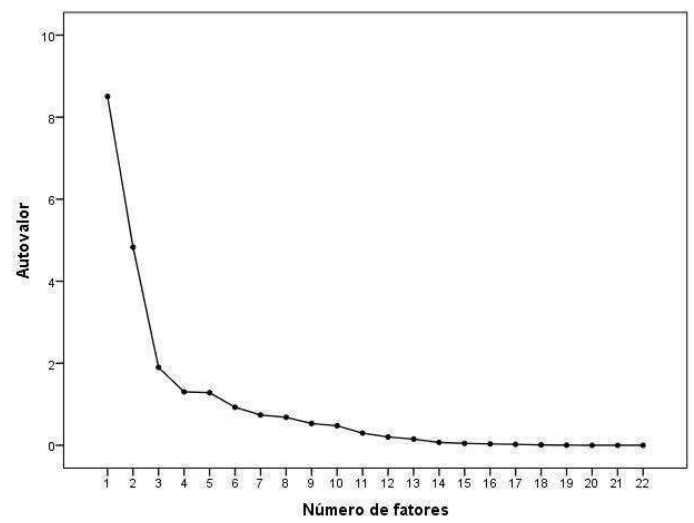

Figura 3. Scree plot para determinar a quantidade de fatores

descresce até estabilizar-se, o que mostra que o aumento do número de fatores não melhorará a variabilidade.

Com tais resultados, buscou-se identificar quais variáveis mais influenciaram em cada fator, ou seja, aquelas que possuem maiores cargas fatoriais. Dessa forma, foi possível nomear cada fator conforme os rótulos de componentes latentes da Tabela 2.

Componentes Latentes

\begin{tabular}{lll}
\hline Fator & Características principais & Componente Latente \\
\hline 1 & opmais $(+)$, opmult $(*)$, opmenos $(-)$ & Operação Aritmética \\
2 & ccase, cbreak, celse, opmaior $(>)$ & Seleção, comparação \\
3 & opmenor $(<)$, opatrib $(=)$ & Comparação, Atribuição \\
4 & cscanf & Entrada de dados \\
5 & opmenorigual $(\geq)$, cdo & Comparação, Repetição \\
\hline
\end{tabular}

Tabela 2. Análise de componentes latentes da Base-B

\subsection{Seleção do Conjunto de Treino}

O Clustering em Grafo foi aplicado nas bases experimentais em dois passos. Primeiro, obtivemos do processo de clustering dois conjuntos: o de amostras heterogêneas (nãoclusterizadas) e o de amostras homogêneas (clusterizadas com similaridade acima de $70 \%$ ). Segundo, se o processo anterior não gerasse um número satisfatório de amostras heterogêneas para formar o treino do classificador, realizamos o re-clustering informando ao algoritmo de clustering para particionar a base em dois clusters. O cluster mais heterogêneo desse particionamento é o cluster selecionado para treinar o classificador.

$\mathrm{Na}$ experimentação do método, a Base-A, sendo maior, foi clusterizada no primeiro passo. Já a Base-B, por não formar um conjunto heterogêneo no primeiro passo, foi dividida em dois clusters no segundo passo. 


\section{CBIE-LACLO 2015}

Anais do XXVI Simpósio Brasileiro de Informática na Educação (SBIE 2015)

A Figura 4 apresenta os gráficos dos clusters 0 e 1 formados pela aplicação do Clustering em Grafo na Base-B. Nesses gráficos, cada linha contém o rótulo da representação vetorial de um exercício de programação e seu score de classe. As colunas são os cincos fatores ou componentes latentes da Base-B gerados pela análise fatorial. A cor vermelha indica valores positivos, a cor verde, valores negativos e a cor branca, valor nulo. Quanto mais intensa é a cor, maior é o valor do seu fator.

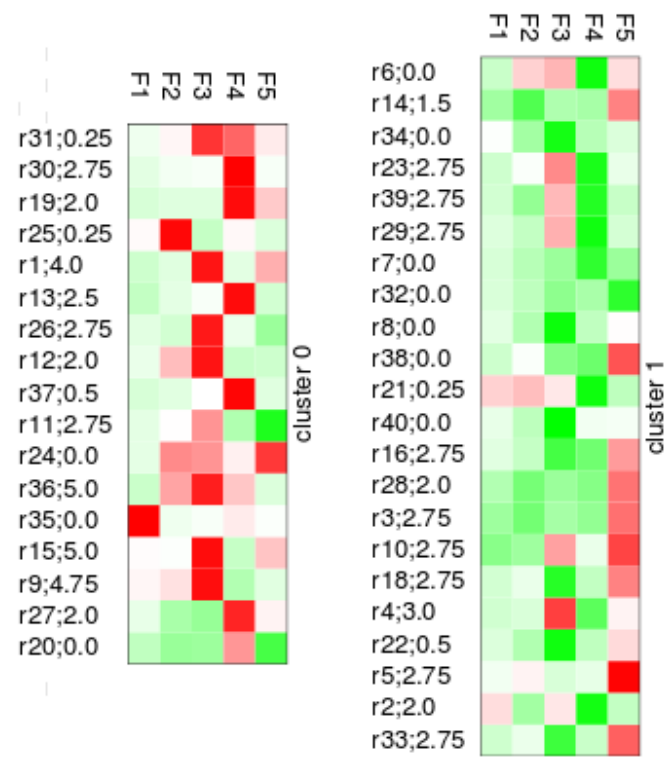

Figura 4. Clustering em Grafo da Base-B

Nos gráficos de Clustering em Grafo da Figura 4, observa-se que a cor vermelha predomina no Cluster $O$ e é mais presente entre os fatores $F_{i}$, o que significa que o Cluster 0 representa melhor a diversidade das componentes latentes para formar o treino do classificador de perfis. Uma confirmação disso está na variedade de scores de classes nas linhas do Cluster 0 e nos resultados da Base-B apresentados na Subseção 4.6.

\subsection{Classificação de Perfis}

A classificação de perfis foi realizada por modelos de regressão linear para obter valores próximos dos scores de classes. Esses modelos foram treinados com as amostras heterogêneas geradas no passo 1 ou com o cluster mais heterogêneo gerado no passo 2 do processo de Clustering.

\subsection{Resultados}

A Tabela 3 apresenta os resultados de aplicação do método de seleção não-aleatória de treino de classificação na Base-A. Sendo a Base-A uma base grande, o algoritmo de Clustering em Grafo formou o conjunto de treino com um número razoável de amostras não clusterizadas, isto é, com as amostras com maior diversidade de componentes latentes.

Os resultados da Base-A nas colunas $s A F$ e $c A F$ da Tabela 3 indicam a aplicação do método sem e com a análise fatorial, respectivamente. A linha Qtd.Treino/Teste informa, respectivamente, quantas amostras de cada base foram selecionadas para treinar o modelo de classificação e quantas amostras o modelo classificou automaticamente. 


\section{CBIE-LACLO 2015}

Anais do XXVI Simpósio Brasileiro de Informática na Educação (SBIE 2015)

Para avaliar o erro de predição de scores da Base-A e da Base-B, utilizamos as medidas de Erro Médio, Erro Mínimo e Erro Máximo. Nas tabelas 3 e 4, quanto menor os valores dessas medidas, melhor é o desempenho do classificador.

\begin{tabular}{lll}
\multicolumn{3}{c}{ Classificação de Perfis - Base-A } \\
\hline Avaliação & sAF & cAF \\
\hline Qtd. Treino/Teste & $53 / 47$ & $\mathbf{3 6 / 6 4}$ \\
\hline Predição de scores & & \\
Erro Médio & 0.8000 & $\mathbf{0 . 7 9 5 4}$ \\
Erro Mínimo & 0 & 0.0200 \\
Erro Máximo & 2.3000 & $\mathbf{1 . 7 8 0 0}$ \\
\hline
\end{tabular}

Tabela 3. Resultados de classificação de perfis - Base-A

Através dos valores em negrito na Tabela 3, podemos observar que as medidas de Erro Médio e Erro Máximo de predição diminuíram com a aplicação da análise fatorial.

A Entropia e a Pureza são medidas de avaliação da homogeneidade de clusters. Na Tabela 4, apresentamos a Entropia e a Pureza dos dois clusters (0 e 1) formados a partir da Base-B. Quanto maior a Entropia e menor a Pureza, mais heterogêneo é um cluster, isto é, maior é a sua diversidade de características.

Classificação de Perfis - Base-B

\begin{tabular}{lllll}
\hline & \multicolumn{2}{c}{ Cluster 0 } & \multicolumn{2}{c}{ Cluster 1 } \\
\hline Avaliação & sAF & cAF & sAF & cAF \\
\hline Qtd. Treino/Teste & $28 / 11$ & $\mathbf{1 7 / 2 2}$ & $11 / 28$ & $22 / 17$ \\
\hline Avaliação de Clusters & & & & \\
Entropia & 0.782 & $\mathbf{0 . 7 8 7 0}$ & 0.220 & 0.497 \\
Pureza & 0.429 & 0.5880 & 0.909 & 0.545 \\
\hline Predição de scores & & & & \\
Erro Medio & 1.6990 & $\mathbf{0 . 9 0 2 2}$ & 2.7142 & 1.9111 \\
Erro Minimo & 0 & 0 & 0 & 0.25 \\
Erro Maximo & 5.000 & $\mathbf{1 . 9 2 0 0}$ & 5 & 4.75 \\
\hline
\end{tabular}

Tabela 4. Resultados de classificação de perfis da Base-B

Na Tabela 4, os resultados da Base-B, que é a base mais difícil, evidenciam que a combinação da análise fatorial com o Clustering em Grafo para selecionar as amostras com maior diversidade de componentes latentes melhoram os resultados de classificação de perfis. Observa-se que, na Tabela 4, o Cluster 0, que, pelas medidas de Entropia e Pureza, é o mais heterogêneo, apresenta melhores resultados do que o Cluster 1.

Com a análise fatorial, apenas com 17 amostras de treino, o Cluster 0 apresentou os melhores resultados de predição para classificar 22 amostras. Sem a análise fatorial, o número de amostras de treino aumentaria para 28 para classificar 11 amostras.

Em relação ao trabalho de [Oliveira et al. 2014], melhoramos os resultados da Base-A ao reduzir o erro máximo de classificação e a quantidade de amostras de treino necessárias para treinar o modelo de classificação. Além disso, o novo método tem a vantagem de realizar uma seleção não-aleatória de amostras de treino conforme a diversidade das componentes latentes.

Esses resultados nos levam a concluir que a combinação da análise fatorial com o Clustering em Grafo para selecionar as amostras mais heterogêneas da Base-A ou o 


\section{CBIE-LACLO 2015}

Anais do XXVI Simpósio Brasileiro de Informática na Educação (SBIE 2015)

cluster mais heterogêneo da Base-B (Figura 4), melhora os resultados de classificação de perfis reduzindo o erro de predição dos scores de classes.

\section{Considerações Finais}

Este trabalho apresentou um método de seleção não-aleatória de treino para classificação de perfis de estudantes de programação. Os resultados mostram que a combinação das técnicas de análise fatorial e de Clustering em Grafo, representando a diversidade de componentes latentes, melhora os resultados de classificação perfis.

Como trabalhos futuros a partir deste, sugerimos que a estratégia de seleção nãoaleatória de treino de modelos de classificação seja avaliada em outros sistemas de aprendizagem supervisionada como, por exemplo, os sistemas de recomendação.

Os estudos apresentados neste trabalho representam uma passo importante para melhorar a aprendizagem de modelos de classificação de perfis de estudantes. Dessa forma, ao selecionar um número menor de amostras representando a diversidade de perfis, reduzimos esforços de professores na atribuição de scores às amostras de exercícios de programação que formarão o conhecimento do classificador.

Já para a prendizagem de programação, o método proposto integrado ao sistema PCodigo de [Oliveira et al. 2015] oferece possibilidades de feedbacks mais imediatos para estudantes e uma prática de programação melhor assistida por professores.

\section{Referências}

Anderson, T. W. (1984). An Introduction to Multivariate Statistical Analysis. Wiley, New York, NY, second edition.

Karypis, G. (2003). Cluto - a clustering toolkit. Dept. of Computer Science, University of Minnesota.

Morrison, D. F. (1990). Multivariate Statistical Methods. McGraw-Hill, third edition.

Moussiades, L. and Vakali, A. (2005). Pdetect: A clustering approach for detecting plagiarism in source code datasets. The computer journal, 48(6):651-661.

Oliveira, M., Monroy, N., Zandonade, E., and Oliveira, E. (2014). Análise de componentes latentes da aprendizagem de programação para mapeamento e classificação de perfis. In Anais do Simpósio Brasileiro de Informática na Educação (SBIE 2014), volume 25, pages 134-143.

Oliveira, M., Nogueira, M., and Oliveira, E. (2015). Sistema de apoio à prática assistida de programação por execução em massa e análise de programas. In CSBC 2015 Workshop de Educação em Informática (WEI), Recife-PE.

Oliveira, M. and Oliveira, E. (2015). Abordagens, práticas e desafios da avaliação automática de exercćios de programação. In CSBC 2015 - DesafIE 2015, Recife-PE.

Pieterse, V. (2013). Automated assessment of programming assignments. In Proceedings of the 3rd Computer Science Education Research Conference on Computer Science Education Research, CSERC '13, pages 4:45-4:56, Open Univ., Heerlen, The Netherlands, The Netherlands. Open Universiteit, Heerlen.

Schaeffer, S. E. (2007). Survey: Graph clustering. Comput. Sci. Rev., 1(1):27-64. 